\title{
Factores Determinantes de la Conducta Intraemprendedora en Pequeñas y Medianas Empresas (PyMes) de la Región Coquimbo en Chile
}

\author{
Segundo R. Cabana, Hector J. Narea y Ricardo D. Orrego \\ Universidad de La Serena, Facultad de Ingeniería, Dpto. de Ing. Industrial, Casilla 554, La Serena - Chile \\ (e-mal: rcabana@userena.cl, hnarea@userena.cl,rorrego@userena.cl)
}

Recibido Nov. 21, 2017; Aceptado Feb. 5, 2018; Versión final Mar. 7, 2018, Publicado Ago. 2018

\begin{abstract}
Resumen
Esta investigación tiene como objetivo analizar los factores organizacionales que influyen en la conducta intraemprendedora de los trabajadores, tomando como población objetivo la fuerza laboral representada por una muestra de 392 personas. Las pequeñas y medianas empresas de la Región de Coquimbo en Chile se encuentran en un escenario competitivo que las motiva a tomar decisiones para mejorar sostenidamente la innovación y productividad. Se presenta un modelo cuyos resultados se comparan con aquellos de métodos de ecuaciones estructurales basado en covarianzas. Se determinó que la conducta intraemprendedora se genera a través del apoyo de la dirección, autonomía en el trabajo, las recompensas y la incertidumbre en el puesto de trabajo, factores que explican en un $62 \%$ la varianza de la identificación organizacional que tiene el trabajador con su empresa. Esta, a su vez, explica en un $51 \%$ la varianza de la conducta intraemprendedora.
\end{abstract}

\section{Determinant Factors of Intrapreneurial Behavior in Small and Medium Size Enterprises (SMEs) of the Coquimbo Region in Chile}

\begin{abstract}
The objective of this research is to analyze the organizational factors that influence the intrapreneurial behavior of workers, taking as a target population the workforce represented by a sample of 392 people. The small and medium enterprises of the Coquimbo Region in Chile are in a competitive scenario that motivates them to make decisions to improve innovation and productivity. A model is presented and the results are compared with those of structural equation methods based on covariances. It was determined that intrapreneurial behavior is generated through the management support, autonomy at work, rewards and uncertainty in the workplace, factors that explain $62 \%$ of the variance of organizational identification the worker has his company. This, in turn, explains by $51 \%$ the variance of intrapreneurial behavior.
\end{abstract}




\section{INTRODUCCIÓN}

El mundo de los negocios ha revolucionado a tal punto que, para sobrevivir a los desafíos disruptivos de los mercados, las Pymes, deben estar en constante innovación. No solo basta con que este sector empresarial ofrezca una variada gama de productos y servicios, sino que estos tienen que ser de valor y responder satisfactoriamente a las necesidades expresadas y no expresadas por sus clientes, con una propuesta de valor que instale barreras de entradas sostenibles y sustentables en su industria. Si hay un problema que preocupa a las empresas, es el agotamiento de sus modelos de negocio que, poco a poco, van dando síntomas de estancamiento en dar ofertas de valor al mercado (Ahmedova, 2015). Así es necesario estratégicamente para las organizaciones, internalizar el valor que les puede aportar potenciar una Conducta intraemprendedora (IB) en su fuerza laboral, para poder acceder a nuevos mercados no atendidos, entregar productos y/o servicios con un valor agregado para los clientes, gracias al uso de estrategias que contribuyen a la diferenciación y, por último, aumentar la fidelización de los colaboradores (Antoncic y Hisrich, 2001).

Existe un consenso global acerca de la importancia de las pymes como actores claves para incrementar el crecimiento económico y lograr que Chile pase de una economía centrado en la eficiencia a una economía centrado en la innovación. En esta evolución invertir en el desarrollo de una conducta intraemprendedora en la fuerza laboral de las Pymes es imprescindible, y lograrlo permitirá reducir las asimetrías que por décadas aún están presentes en la economía Chilena (Ver Fig. 1). El desarrollo económico depende esencialmente de la capacidad para introducir innovaciones al interior de la base productiva y tejido empresarial de un territorio, y es preciso subrayar que la estrategia de desarrollo tiene su importancia en términos de empleo, basados en factores económicos, sociales, culturales y territoriales (Barragán y Ayaviri, 2017). Es importante destacar que solo un $4,6 \%$ de las empresas logra colocar sus productos en los mercados internacionales, ventaja poco aprovechada por las empresas de menor tamaño. En efecto, mientras en las grandes empresas un $28,4 \%$ de ellas exporta, solo un $5,7 \%$ de las pymes lo hace. Además, en este último grupo de empresas, la mitad exporta a través de un intermediario. Por lo tanto, existe todavía un gran espacio para aprovechar las posibilidades que tiene nuestra profunda integración con el resto del mundo (MEFT, 2015).

Por lo anterior, Chile y particularmente la Región de Coquimbo, deben asumir el desafío de aumentar el valor de los productos y servicios comercializados por las empresas Pymes, donde la conducta intraemprendedora de la fuerza laboral, influirá en aumentar el valor agregado de sus exportaciones, pues actualmente el $84 \%$ corresponde a productos básicos, además impactará en reducir la brecha de productividad del trabajador entre Chile (22 US\$/hr) y la Organización para Cooperación y Desarrollo Económico (49 US\$/hr) (Ministerio de Economía, Fomento y Turismo de Chile, 2016). El desarrollo económico de las naciones y por consiguiente el bienestar general de todos sus habitantes, depende fundamentalmente de las actividades desarrolladas por sus empresas, las cuales están sometidas a fuertes presiones tanto de los competidores locales como internacionales. Este estado de las cosas obliga a estas organizaciones a innovar en sus estrategias de gestión para alinearlas con estas nuevas exigencias, siendo una de sus alternativas capitalizar el compromiso, capacidad, ingenio y creatividad de su talento humano hacia el diseño de una nueva filosofía organizacional (Rubio, 2015).

\section{CONDUCTA INTRAEMPRENDEDORA}

Ming (2013), afirma que "el intraemprendimiento se convierte hoy en día en el más importante proceso en la gestión estratégica, en particular, en la capacidad de las compañías para incrementar la rentabilidad a través del tiempo". Demirci (2013) define el intraemprendimiento como una de las estrategias clave de las organizaciones de hoy, buscando adquirir o sostener la ventaja competitiva en los mercados globales. Sheshadri y Tripathy (2006) lo definen como el proceso a través del cual un individuo o un grupo de individuos en asociación con una organización existente, crean una nueva organización o instigan la renovación o innovación al interior de la organización.

La innovación es una habilidad competitiva para las organizaciones que logran combinar y articular aspectos de la cultura, la organización empresarial, la investigación y desarrollo, la transferencia de tecnología acompañada de un enfoque hacia la especialización y por supuesto la orientación motivadora hacia el talento humano como impulsor o dinamizador de los cambios (Simanca et al., 2016). Las prácticas innovadoras en las organizaciones conducen a generar las ventajas competitivas sostenibles. Como estrategia de desarrollo empresarial, la innovación no sólo está orientada a la generación de nuevos productos y procesos, sino también, a la adaptación y mejora de tecnologías y a la adopción de cambios en la cultura empresarial, en fin, a la introducción permanente de cambios que permitan incrementar la productividad y competitividad de las empresas (Hernández et al., 2017). 
La capacidad de innovar representa hoy, el principal determinante del incremento de la productividad y la competitividad en las empresas y, por ende, de los países y regiones, siendo los resultados de esa capacidad la principal fuente de ventajas competitivas dinámicas (Cabarcas et al., 2015). La innovación desarrollada por la actividad intraemprendedora podría considerarse como una de las capacidades dinámicas, que representan la habilidad de la firma para integrar, construir y reconfigurar las competencias internas y externas en todos los ámbitos de la organización, y dirigirla hacia los entornos cambiantes.

El propósito de este estudio es identificar y analizar las principales variables que influyen en la generación de una conducta intraemprendedora en las empresas Pymes de la Región de Coquimbo, para así contribuir en la instalación de prácticas empresariales que posibiliten a las Pymes chilenas, romper las señales significativas de estancamiento del valor de sus productos y servicios, considerando la línea base detallada previamente, donde la oferta de este segmento empresarial está perdiendo valor en su mercado o bien no existe una propuesta de valor innovadora, que le dé una diferencial sustentable a largo plazo, lo que es consistente con lo expresado por el Ministerio de Economía, Fomento y Turismo de Chile (2016).

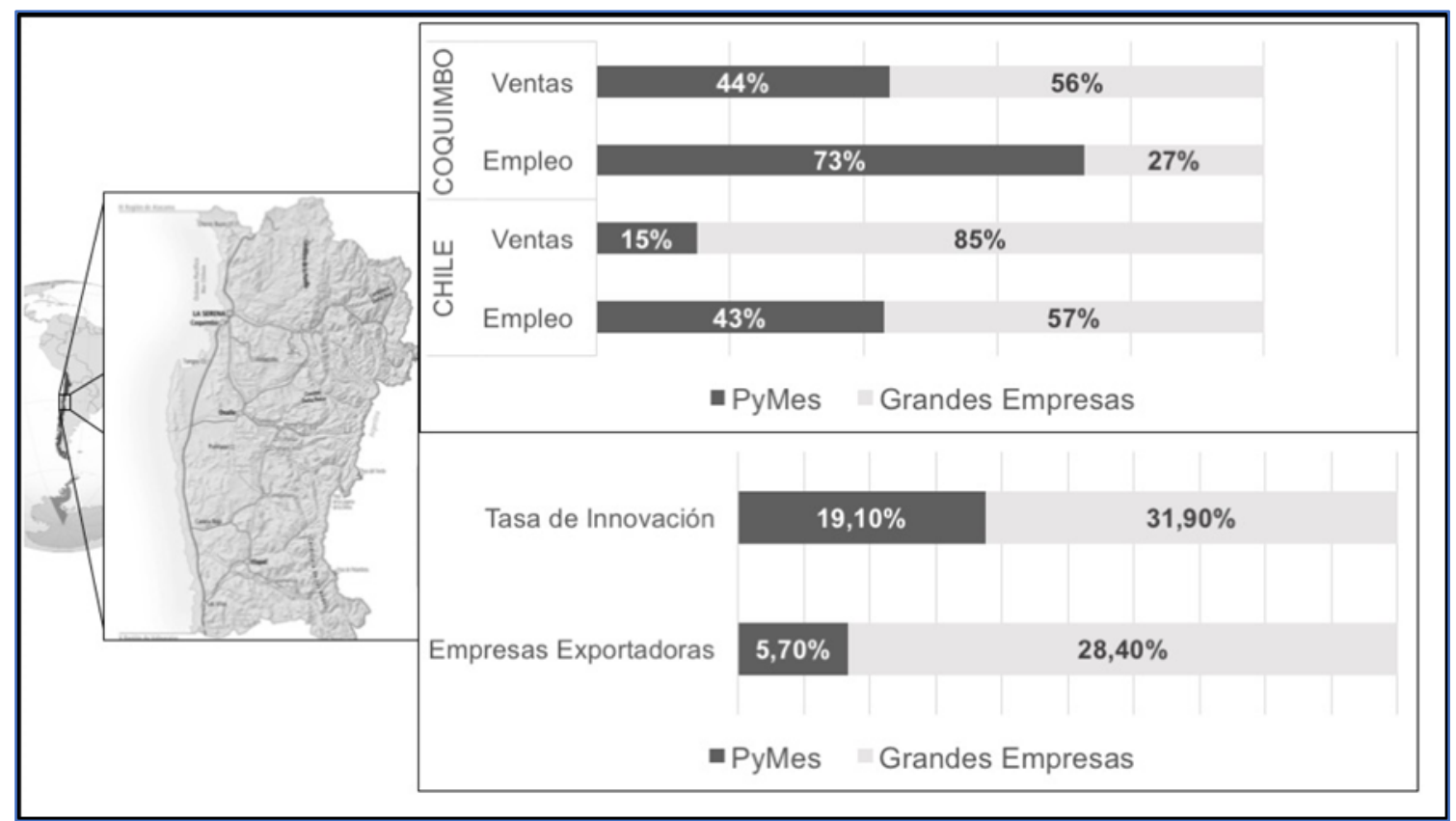

Fig. 1: Línea Base de Participación por factor de PyMes y Gran empresa a nivel de Chile y Región de Coquimbo.

\section{COMPONENTES DE LA CONDUCTA INTRAEMPRENDEDORA}

El estudio de Ağca et al. (2012), tiene en cuenta los puntos de vista de la orientación emprendedora y el espíritu empresarial corporativo, y clasifica cinco dimensiones de la IB: (1) la capacidad de innovación, (2) nuevas aventuras de negocio, (3) la auto-renovación o renovación estratégica, (4) la asunción de riesgos y (5) proactividad, que incluye agresividad competitiva. Moriano et al. (2009), propone un modelo que explica la IB de los miembros de la organización como el resultado de la mediación de la identificación con la organización sobre los efectos de estos factores organizacionales. De esta forma, plantea que el apoyo de la dirección, la libertad en el trabajo, las recompensas y el tiempo disponible generan una influencia positiva sobre identificación que a su vez influirá positivamente en el desarrollo de la IB de los miembros de la organización, mientras que la incertidumbre en las tareas tiene una influencia negativa.

\section{Hipótesis 1: El apoyo de la dirección, influye directa y positivamente en la identificación organizacional}

Los líderes empresariales de la innovación no deben mantenerse al nivel de la creación de productos únicamente, sino que deben saltar a influir en la esfera de la alta gerencia, así aumentarán las probabilidades de éxito en la tarea de implementar una cultura de innovación. La identificación organizacional debe ser vigilada y administrada al nivel de la más alta gerencia, siendo la innovación una herramienta y no un fin en sí misma. La percepción de respaldo por parte de la organización que tiene el trabajador o la confianza en el supervisor, hacen que la persona sienta un mayor nivel de identificación grupal. Así, por ejemplo, la relevancia de la alta dirección en la identificación organizacional radica en que los directivos tienen un rol fundamental al momento de formular las políticas de la empresa, asignar recursos, diseñar programas y establecer y mantener una cultura corporativa sustentable (Arancibia et al., 2015). 
El estudio de Forsyth y Mason (2017), plantea que existe una asociación positiva entre la identificación profesional de los trabajadores con el tipo de liderazgo aplicado a los participantes. La misma asociación fue demostrada para la identificación del equipo y liderazgo. Los hallazgos ponen en relieve el importante vínculo entre la identificación de grupos y los estilos de liderazgo, lo que sugiere que las estrategias que promueven fuertes identificaciones profesionales y de equipos tienden a ser propiciadas por aquellas jefaturas que aplican un liderazgo centrado en el apoyo. Líderes con un alto nivel de apoyo en sus seguidores logran que estos estén más satisfechos, sean más eficaces y dediquen un esfuerzo extra en su lugar de trabajo (Barbosa, 2013).

\section{Hipótesis 2: La autonomía en el trabajo influye directa y positivamente en la identificación organizacional.}

Existe una relación positiva entre la delegación, la decisión mediante empoderamiento, decisiones en conjunto y el comportamiento de los empleados. Se demostró que el liderazgo participativo desempeña un papel esencial en el comportamiento global del empleado (Fahed-Sreih 2012). Una alta autonomía, permitirá a los equipos tener una comprensión más clara de los objetivos de la organización, lo que a su vez aumenta el rendimiento del equipo (Gonzalez-Mulé et al., 2016).

De acuerdo a Chávez (2014), dar la capacidad para que los empleados realicen sus actividades, los hará sentir con una mayor libertad para ser creativos en sus métodos de trabajo; si a esto se le agrega la facultad de decisión, las habilidades laborales tenderán a desarrollarse y el compromiso por hacer efectivamente el trabajo se volverá una constante en las funciones. Es importante considerar que la confianza y motivación, deberán ser los factores que den a la gente el impulso para tener mejores resultados, porque un ambiente organizacional de apoyo impulsará el involucramiento de la gente en el trabajo. Cabe destacar que a pesar de los beneficios que aporta esta componente de la conducta intraemprendedora, en muchos casos los gerentes no están dispuestos a ofrecer a los empleados mayores niveles de autonomía y los beneficios asociados, porque su función principal sigue siendo el "control del trabajo".

\section{Hipótesis 3: El empleo de recompensas influye directa y positivamente en la identificación organizacional.}

Nunca se deja de experimentar la necesidad de sentirse valorado y recompensado por el trabajo, incluso cuando se es independiente y autosuficiente. Desde una perspectiva relacionada con la calidad de vida en el trabajo, las recompensas deben de ser suficientes para satisfacer las necesidades del personal. De no ser así, los empleados no estarán contentos ni satisfechos en la organización, ya que tienden a comparar los sistemas de recompensas con los de otras empresas. Asimismo, quieren que las recompensas sean otorgadas de manera equitativa, por lo cual comparan sus recompensas, basadas en el rendimiento, que refuerzan los logros significativos e incentivan el enfrentarse a nuevos retos con las recibidas por otros miembros de la organización (Kuratko et al., 2005).

La remuneración y los incentivos contribuyen a la implementación de las estrategias porque condicionan la conducta de las personas y del grupo, pues lo motivan a que dirijan su desempeño hacia las metas de la organización, dado que contribuyen con la identificación organizacional del personal (García et al., 2012). Los niveles de motivación dentro del lugar de trabajo tienen un impacto directo en la productividad de los empleados, los trabajadores que están motivados y entusiasmados con su trabajo cumplen con sus responsabilidades al máximo de su capacidad y aumentan la productividad como resultado (Chaitanya, 2014). La distribución de valor al personal aumenta su motivación y satisfacción, contribuyendo con mayor eficiencia y eficacia al logro de los objetivos organizacionales, lo cual implica un aporte al incremento en el valor económico de la empresa (Medina et al., 2008).

Hipótesis 4: El tiempo disponible influye directa y positivamente en la identificación organizacional.

La gestión del tiempo es una herramienta que permite manejar y disponer plenamente del tiempo de trabajo, evitando en lo posible toda interrupción que no aporte nada a los objetivos de la organización, que por cierto también implica satisfacer los objetivos personales y así contribuir con la identificación organizacional (Mengual et al., 2012). Actualmente, además de las recompensas tangibles, es imprescindible la búsqueda de motivaciones emocionales para generar la mayor pertenencia y productividad posible (Sánchez, 2014).

\section{Hipótesis 5: La incertidumbre influye directa y negativamente en la identificación organizacional.}

Sánchez (2014) indica que la desorientación de los empleados cuando no saben muy bien qué funciones deben realizar, afecta la relación con otros compañeros, con los superiores y con la organización, es decir daña la identificación con la misma. Esta incertidumbre acerca de las tareas dentro de la empresa, puede llevar a los empleados a desarrollar estrés, lo que lleva a éstos, en ocasiones, a formular estrategias para reducirlo llevándolo a un alejamiento psicológico de la situación que causa el estrés, con menos 
compromiso, menos satisfacción en el trabajo y una pronunciada intención de dejar la organización (Chirumbolo y Hellegren, 2003). Entregar definiciones claras sobre las tareas es la manera de optimizar su calidad de vida en el trabajo (García et al., 2014).

Hipótesis 6: La identificación organizacional influye directa y positivamente en la conducta intraemprendedora. Una empresa que disponga de empleados altamente identificados podrá disfrutar de mayor compromiso y lealtad, sus miembros estarán más satisfechos, menos estresados, con mayor autoestima y motivación para contribuir con ideas o acciones innovadoras en beneficio de la empresa, participando del éxito de la organización a la que pertenecen (Topa y Morales, 2006). De esta manera se podrán reconocer obstáculos que le permitan aprender de modo que, con un enfoque proactivo, tanto las personas como la organización, promueven su cambio continuo y gestionan sus conocimientos basados en la realidad, logrando así, una transformación y desarrollo de su inteligencia y capacidad creativa (Chávez, 2014). Esta implicación e identificación son rasgos claramente definitorios del intraemprendedor, ya que éste se identifica con la empresa, sus valores y cultura, y por eso siente que tiene que aportar un valor más allá de su trabajo diario. Uno de los beneficios de las actividades llevadas a cabo por el intraemprendedor es la innovación, que puede considerarse como una de las capacidades dinámicas, que representan la habilidad de la firma para integrar, construir y reconfigurar las competencias internas y externas en todos los ámbitos de la organización, y dirigirla hacia los entornos cambiante (Frías y Véliz, 2013).

Considerando todas las relaciones anteriores, se plantea un modelo causal que busca representar las variables que influyen en la Conducta Intraemprendedora de trabajadores de pequeñas y medianas empresas en la Región de Coquimbo-Chile (Fig. 2).

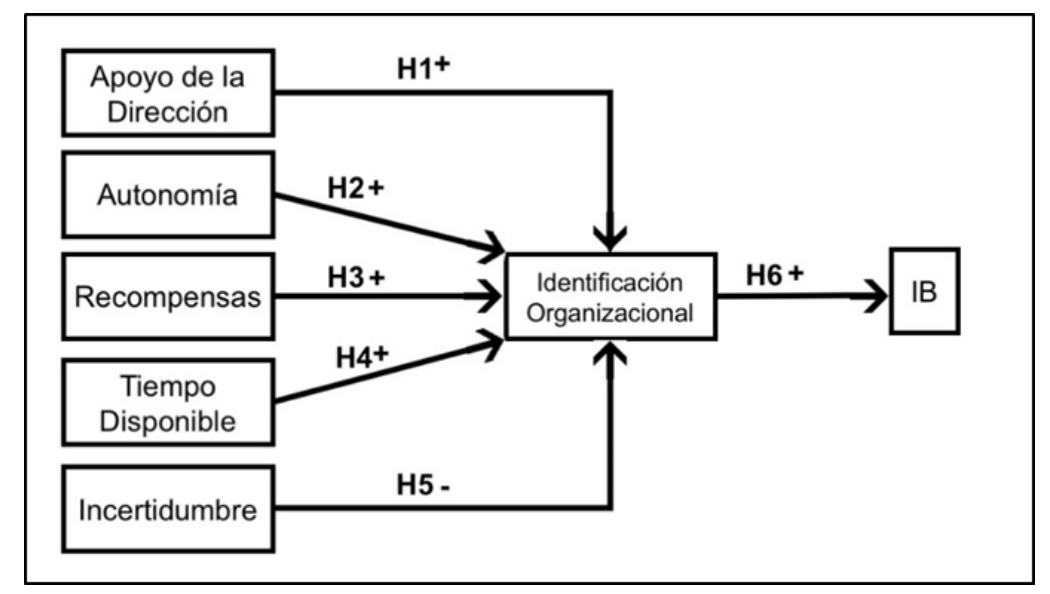

Fig. 2: Modelo Causal Conducta Intraemprendedora

\section{METODOLOGÍA}

El método empleado para recoger la información fue a través de una encuesta estructurada aplicada a los trabajadores. La ficha técnica de la investigación se presenta en la Tabla1. El instrumento de evaluación utilizado se basa en la encuesta propuesta por Moriano et al., (2009) ajustada al contexto regional de Coquimbo, siendo validada por expertos en intraemprendimiento del sector privado. La muestra se integra por $58.4 \%$ de hombres y $41.6 \%$ de mujeres, el rango de edad predominante es entre 26 y 35 años (37.5\%), mientras que entre 35 y 45 años abarcan el 18.5\%. Del total de los encuestados, el $41 \%$ corresponden a cargos de jefaturas, gerencias o supervisores, teniendo un $58 \%$ de trabajadores profesionales. Finalmente, un $35.4 \%$ posee una antigüedad en la empresa entre 1 y 5 años, mientras que aquellos con 16 o más años de antigüedad en la empresa corresponden al $20.3 \%$.

\section{Análisis de Fiabilidad Individual de los Indicadores}

Para determinar la fiabilidad individual de los indicadores fue necesario calcular las cargas factoriales de cada uno, lo que es ejecutable pues el índice de Kaiser-Meyer-Olkin (KMO) fue superior a 0.6 para cada uno de los constructos y la prueba de esfericidad de Barlett (PEB) fue significativa $(p<0.05)$ (Espinoza, Sanhueza, Ramírez, \& Sáez, 2015) (Tabla 2). Para esto se utilizó el software IBM SPSS Statisctics 21, utilizando el método de extracción de Máxima Verosimilitud y el método de rotación VARIMAX. Se aceptarán las cargas factoriales con valores superiores a 0.6 (Moriano et al., 2009). Bajo este análisis se eliminaron los indicadores AD1, AD2, AT1, AT2, AT3, ER3, TD3, TD6, IN5 el resto de los indicadores se consideran aceptables y significativos al 95\% (Tabla 2). 


\section{Análisis de Fiabilidad del Constructos}

Para evaluar la fiabilidad del constructo se debe tener en cuenta la consistencia interna, la que exige que el Alpha de Cronbach sea mayor a 0.7 (Hameed y Abdul, 2014). Junto a lo anterior se realiza el cálculo de la fiabilidad compuesta (IFC) la cual tiene en cuenta las interrelaciones de los constructos; y tiene como valor mínimo 0.7(Hameed y Abdul, 2014). Los resultados (Tabla 2) indican que existe una consistencia interna de cada uno de los ítems que componen los constructos.

Tabla 1: Ficha Técnica de la Investigación.

\begin{tabular}{|l|l|}
\hline \multicolumn{1}{|c|}{ Tipo de muestreo } & \multicolumn{1}{c|}{ Muestreo Aleatorio } \\
\hline Nivel de confianza & $95 \% ; \mathrm{z}=1,96 ; \mathrm{p}=\mathrm{q}=0,5$ (5\% error y $95 \%$ de confiabilidad). \\
\hline Tamaño muestral & 392 trabajadores \\
\hline Unidad de análisis & Región de Coquimbo, Chile \\
\hline Método de recogida de la información & Cuestionario presencial y online de 58 preguntas \\
\hline Tipo de pregunta encuesta & Politómicas tipo Likert (1 a 5) puntuaciones o categorías y de selección. \\
\hline Fecha de realización del trabajo de campo & Entre diciembre de 2015 y mayo de 2016 \\
\hline
\end{tabular}

\section{Validez Convergente y Discriminante}

Para continuar, se evalúa la validez de las escalas utilizadas (validez convergente), para lo cual se analiza la varianza extraída media (AVE). Se recomienda que esta sea superior a 0,5 con lo que se establece que más del $50 \%$ de la varianza del constructo se debe a sus indicadores (Hameed y Abdul, 2014) (Echeverri et al., 2018). Todos los constructos entregan un AVE superior al mínimo esperado, por lo que se comprueba la validez convergente de éstos (Tabla 2). Finalmente, la validez discriminante indica en qué medida un constructo es diferente a los otros que conforman el modelo. Una forma de comprobar este criterio de validación, es demostrar que las correlaciones entre los constructos son más bajas que la raíz cuadrada de la varianza extraída media AVE (Hameed y Abdul, 2014). Como se observa en la Tabla 3, todos los valores cumplen para la validez discriminante.

Tabla 2: Resultados del análisis estadístico: carga factorial, KMO, alpha de cronbach, IFC y AVE

\begin{tabular}{|c|c|c|}
\hline Constructo & Indicador & Carga Factorial $(\lambda)$ \\
\hline $\begin{array}{l}\text { Innovación } \\
K M O=0,773 \\
\text { Alpha de Cronbach }=0,820 \\
\text { IFC }=0,953 \\
\text { AVE }=0,716\end{array}$ & $\begin{array}{l}\text { 11: Realizar Intraemprendimientos } \\
\text { I2: Desarrollar nuevos procesos } \\
\text { I3: Innovar en las tareas } \\
\text { 14: Nuevas formas de hacer las cosas }\end{array}$ & $\begin{array}{l}0,84 \\
0,81 \\
0,86 \\
0,86\end{array}$ \\
\hline $\begin{array}{l}\text { Asunción de Riesgos } \\
K M O=0,713 \\
\text { Alpha de Cronbach }=0,796 \\
I F C=0,924 \\
A V E=0,738\end{array}$ & $\begin{array}{l}\text { AR1: Asumir riesgos no calculados en tareas } \\
\text { AR2: Asumir riesgos no calculados en actividades } \\
\text { AR3:Asumir riesgos calculados }\end{array}$ & $\begin{array}{l}0,84 \\
0,86 \\
0,86\end{array}$ \\
\hline $\begin{array}{l}\text { Apoyo de la Dirección } \\
K M O=0,951 \\
\text { Alpha de Cronbach }=0,942 \\
I F C=0,948 \\
A V E=0,518\end{array}$ & $\begin{array}{l}\text { AD1: Rapidez en mejorar métodos de trabajo } \\
\text { AD2: Trabajadores desarrollan nuevas metodologías } \\
\text { AD3: Estimulo al desarrollo de ideas } \\
\text { AD4: Conciencia y recepción de ideas } \\
\text { AD5: Promoción de cargo basado en aportes } \\
\text { AD6: Apoyo de la dirección para nuevas ideas } \\
\text { AD7: Libertad para iniciativas de los trabajadores } \\
\text { AD8: Directivos motivan al personal } \\
\text { AD9: Experiencia de los directivos en innovación } \\
\text { AD10: Aporte monetario para nuevas ideas } \\
\text { AD11:Compensaciones por ideas exitosas } \\
\text { AD12: Formas para conseguir apoyo económico } \\
\text { AD13: Motivación a asumir riesgos } \\
\text { AD14: Reconocimientos por iniciativas }\end{array}$ & \begin{tabular}{l|}
0,58 \\
0,56 \\
0,71 \\
0,69 \\
0,69 \\
0,77 \\
0,62 \\
0,69 \\
0,68 \\
0,62 \\
0,72 \\
0,76 \\
0,78 \\
0,78
\end{tabular} \\
\hline
\end{tabular}


Tabla 2 (continuación)

\begin{tabular}{|c|c|c|}
\hline & $\begin{array}{l}\text { AD15: Percepción del riesgo por parte de los directivos } \\
\text { AD16: Apoyo a proyectos experimentales } \\
\text { AD17: Concesión de tiempo para desarrollo de ideas } \\
\text { AD18: Transversalidad de la organización } \\
\text { AD19: Comunicación de aportes }\end{array}$ & $\begin{array}{l}0,72 \\
0,74 \\
0,74 \\
0,67 \\
0,78\end{array}$ \\
\hline $\begin{array}{l}\text { Autonomía } \\
K M O=0,929 \\
\text { Alpha de Cronbach }=0,914 \\
\text { IFC }=0,938 \\
\text { AVE }=0,683\end{array}$ & $\begin{array}{l}\text { AT1: Tomar decisiones propias } \\
\text { AT2: Métodos de trabajo determinados por la organización } \\
\text { AT3: Castigos a los errores } \\
\text { AT4: Decisión propia de cómo hacer el trabajo } \\
\text { AT5: Oportunidades de ser creativo } \\
\text { AT6: Nuevas ideas alienadas a los objetivos de la empresa } \\
\text { AT7: Libertad para utilizar escritorios propias } \\
\text { AT8: Responsabilidad sobre las decisiones } \\
\text { AT9: Posibilidad de utilizar habildiades propias } \\
\text { AT10: Libertad para decidir qué hacer.. }\end{array}$ & $\begin{array}{l}0,58 \\
0,55 \\
0,36 \\
0,82 \\
0,84 \\
0,83 \\
0,86 \\
0,83 \\
0,78 \\
0,84\end{array}$ \\
\hline $\begin{array}{l}\text { Recompensas } \\
\mathrm{KMO}=0,817 \\
\text { Alpha de Cronbach }=0,826 \\
\mathrm{IFC}=0,879 \\
\mathrm{AVE}=0,594\end{array}$ & $\begin{array}{l}\text { RE1: Apoyo de supervisores o jefes } \\
\text { RE2: Incentivos alienados al trabajo } \\
\text { RE3: Responsabilidades adhoc al trabajo realizado } \\
\text { RE4: Reconocimiento al buen desempeño } \\
\text { RE5: Recomendación del trabajador } \\
\text { RE6: Retos Motivadores }\end{array}$ & $\begin{array}{l}0,66 \\
0,71 \\
0,10 \\
0,87 \\
0,84 \\
0,75\end{array}$ \\
\hline $\begin{array}{l}\text { Tiempo Disponible } \\
K M O=0,748 \\
\text { Alpha de Cronbach }=0,702 \\
\text { IFC }=0,796 \\
\text { AVE }=0,51\end{array}$ & $\begin{array}{l}\text { TD1: Carga de trabajo } \\
\text { TD2: Tiempo de ocio } \\
\text { TD3: Tiempo empleado para realizar tareas } \\
\text { TD4: Tiempo de calidad para el trabajador } \\
\text { TD5: Trabajo bajo presión temporal } \\
\text { TD6: Tiempo para idear soluciones empresariales }\end{array}$ & $\begin{array}{l}0,64 \\
0,71 \\
0,50 \\
0,68 \\
0,69 \\
0,59\end{array}$ \\
\hline $\begin{array}{l}\text { Incertidumbre } \\
K M O=0,791 \\
\text { Alpha de Cronbach }=0,812 \\
\text { IFC }=0,866 \\
\text { AVE }=0,519\end{array}$ & $\begin{array}{l}\text { IN1: Procedimientos establecidos } \\
\text { IN2: Reglas y procedimientos documentados } \\
\text { IN3: Dudas sonbre el desempeño esperado } \\
\text { IN4: Incertidumbre en el puesto laboral } \\
\text { IN5: Discusión del desempeño } \\
\text { IN6: Descripción clara del puesto laboral } \\
\text { IN7: Nivel de desempeño laboral esperado }\end{array}$ & $\begin{array}{l}0,67 \\
0,70 \\
0,76 \\
0,67 \\
0,29 \\
0,73 \\
0,78\end{array}$ \\
\hline $\begin{array}{l}\text { Ident. Organizacional } \\
K M O=0,793 \\
\text { Alpha de Cronbach }=0,824 \\
I F C=0,899 \\
A V E=0,69\end{array}$ & $\begin{array}{l}\text { IO1: Sentido de pertenencia con la organización } \\
\text { IO2: Gusto por la organización } \\
\text { IO3: Satisfacción con el equipo de trabajo }\end{array}$ & $\begin{array}{l}0,76 \\
0,89 \\
0,87\end{array}$ \\
\hline
\end{tabular}

Tabla 3: Validez Discriminante

\begin{tabular}{|l|l|l|l|l|l|l|l|}
\hline \multicolumn{1}{|c|}{ Constructo } & 1 & 2 & 3 & 4 & 5 & 6 & 7 \\
\hline 1. Apoyo de la Dirección & 0.719 & & & & & & \\
\hline 2. Autonomía & 0.533 & 0.826 & & & & & \\
\hline 3. Recompensas & 0.692 & 0.574 & 0.771 & & & & \\
\hline 4. Tiempo disponible & 0.573 & 0.731 & 0.803 & 0.663 & & & \\
\hline 5. Incertidumbre & -0.540 & 0.447 & 0.593 & 0.770 & 0.720 & & \\
\hline 6. Identificiación Organizacional & 0.553 & 0.594 & 0.584 & 0.321 & 0.473 & 0.832 & \\
\hline 7. IB & 0.387 & 0.494 & 0.291 & 0.228 & 0.245 & 0.346 & 0.846 \\
\hline
\end{tabular}




\section{RESULTADOS}

Luego de realizar el análisis factorial exploratorio y confirmatorio del modelo propuesto, se procede a utilizar el método de modelo de ecuaciones estructurales, a través del método basado en las covarianzas (MBC), debido a que este método está orientado a la estimación de parámetros, trabaja con indicadores reflectivos y, además, es el que mejor se ajusta a las características del modelo, al tener menos de 100 indicadores se considera un modelo de complejidad pequeña (Figura 3).

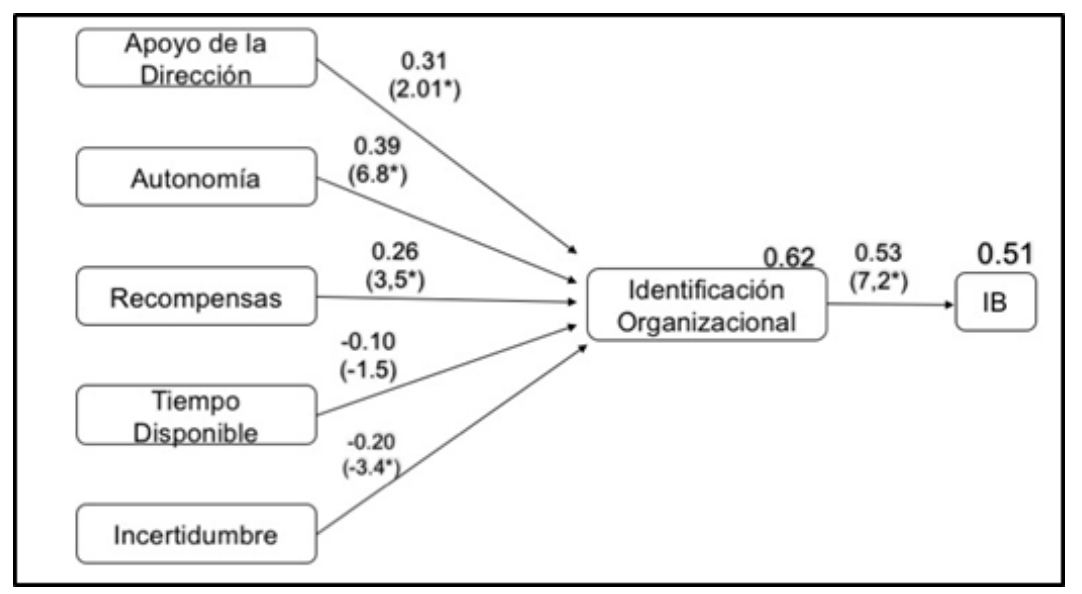

Fig.3: Modelo de Ecuaciones Estructurales

\section{Análisis de Relaciones Causales y Contraste de Hipótesis}

Los resultados indican la existencia de relaciones causales significativas entregadas por el coeficiente estandarizado y por el valor absoluto del ratio crítico de los constructos superior a 1.96 en las hipótesis planteadas inicialmente, por lo que se comprueban y aceptan cinco (5) de las seis (6) hipótesis propuestas (Tabla 4).

Tabla 4: Hipótesis del Estudio

\begin{tabular}{|l|l|l|l|l|}
\hline Hipótesis & \multicolumn{1}{|c|}{ Relación Estructural } & $\begin{array}{c}\text { Coeficiente } \\
\text { Estandarizado }\end{array}$ & $\begin{array}{l}\text { Ratio Crítico } \\
(t \text {-value })\end{array}$ & Contraste \\
\hline H1 & Apoyo de la Dirección $\rightarrow$ Identificación Organizacional & 0,31 & 2,01 & Acepta \\
\hline H2 & Autonomía $\rightarrow$ Identificación Organizacional & 0,39 & 6,80 & Acepta \\
\hline H3 & Recompensas $\rightarrow$ Identificación Organizacional & 0,26 & 3,50 & Acepta \\
\hline H4 & Tiempo disponible $\rightarrow$ Identificación Organizacional & $-0,10$ & $-1,50$ & Rechaza \\
\hline H5 & Incertidumbre $\rightarrow$ Identificación Organizacional & $-0,20$ & $-3,40$ & Acepta \\
\hline H6 & Identificación Organizacional $\rightarrow$ IB & 0,53 & 7,20 & Acepta \\
\hline
\end{tabular}

Bondad de ajuste del modelo

Para determinar la pertinencia del modelo propuesto se utilizaron indicadores de bondad de ajuste, los cuales son indicadores de la pertinencia de una teoría de relaciones entre variables, dada por la correspondencia entre el modelo que la representa y los datos utilizados para probar esa teoría (Gaxiola et al., 2012). Los indicadores estudiados corresponden índice de ajuste comparativo (CFI), índice de ajuste incremental (IFI), índice de error medio cuadrático de aproximación (RMSEA) y residuo cuadrático medio (RMR), los cuales se detallan en la tabla 5, donde se observa que las medidas de bondad de ajuste indican estar dentro de los niveles recomendados, por lo tanto, se puede afirmar que el ajuste global es aceptable.

Tabla 5: Bondad de Ajuste

\begin{tabular}{|c|c|}
\hline Indicador & Valor \\
\hline RMR & 0.092 \\
\hline CFI & 0,844 \\
\hline NFI & 0,765 \\
\hline RMSEA & 0,061 \\
\hline
\end{tabular}




\section{DISCUSIÓN}

El primer aporte relevante de esta investigación, es que se ratifica con confiabilidad estadística que la conducta intraemprendedora de los trabajadores de empresas Pymes en la Región de Coquimbo, Chile, se genera a través de la gestión sistémica de cuatro variables; apoyo de la dirección, autonomía en el trabajo, las recompensas asociadas a los resultados logrados y la incertidumbre en el puesto de trabajo, factores que explican en un $62 \%$ la varianza de la identificación organizacional que tiene el trabajador con su empresa, y ésta a su vez explica en un $51 \%$ la varianza de la conducta intraemprendedora, que es un nivel significativo según Moriano et al. (2009). Con estos resultados se confirma la importancia de la identificación organizacional como variable mediadora entre los factores de la organización y la conducta intraemprendedora de los trabajadores de empresas privadas. Por lo tanto, si el trabajador se identifica con su empresa, se puede afirmar que asumirá riesgos y generará y/o ejecutará nuevas ideas de "negocios" que vayan en beneficio de la organización, dado que estás son las principales componentes de la conducta intraemprendedora, lo que es consistente con lo expresado por Moriano et al. (2009), Frias y Véliz (2013) y, Ağca et al. (2012).

Los resultados univariantes de esta investigación evidencian que las gerencias de las empresas Pymes no gestionan sistémicamente las cuatro variables que influyen en la conducta intraemprendedora, sus niveles se encuentran en un rango bajo ( 41 a $60 \%$ ) a medio (61 a $80 \%)$, según la escala usada en Cabana et al. (2016). Para el caso de apoyo de la dirección los resultados alcanzan un nivel de un $55 \%$, recompensas un $58 \%$, incertidumbre un $65 \%$, autonomía un $62 \%$ e identificación un $53 \%$, generando en su conjunto bajos niveles de intraemprendimiento en las Pymes equivalente a un $42 \%$ efectivo, lo que implica que sólo 4 de cada 10 trabajadores ha ejecutado realmente de iniciativas innovadoras en beneficio de los procesos 0 negocios de la empresa.

Junto a lo anterior, se destacan los resultados del análisis bivariante que permiten obtener la relación que existe entre el género y edad, con la Conducta Intraemprendedora. Bajo el contexto anterior, al realizar el test de Fisher, en ambos casos se rechaza la hipótesis alterna, pues el $\mathrm{F}$ calculado es menor al $\mathrm{F}$ tabular (al $5 \%$ de significancia). Por lo tanto, el nivel de la conducta intraemprendedora de la fuerza laboral de las empresas Pymes, es independiente tanto del género como la edad de los mismos, pues no se evidencian estadísticamente diferencias significativas, resultados que concuerdan con lo expresado por Fernandez et al. (2010), quienes en su estudio revelan mínima influencia del sexo del trabajador en el desarrollo de su actividad laboral, pues los roles y habilidades no son exclusivos de ninguno de los géneros. Esto puede surgir como consecuencia de las nuevas tendencias y demandas organizativas que propician un entorno más favorable hacia la diversidad de género, y por Chiang et al. (2010), quienes plantean que no existen diferencias al segmentar una muestra por la edad en términos de innovación, compromiso y clima organizacional

Con el propósito de complementar el estudio, se llevaron a cabo análisis de dos variantes del modelo, en primer lugar se excluye el constructo tiempo disponible, bajo el criterio que en el modelo puro (Figura 3) no cumplía con el grado de significancia establecido (t-value>1.96). A partir de esto, se ratifica la poca relevancia del tiempo disponible sobre la identificación, pues la varianza explicada de ésta última no varía significativamente $(-2 \%)$. Junto a lo anterior se realizó una segunda variante, en la que no existe la mediación de la identificación organizacional entre los factores y la conducta intraemprendedora, en este caso se puede apreciar que solo los constructos apoyo de la dirección y autonomía tienen un impacto significativo sobre la IB, en conjunto estas variables explican sólo un $34 \%$ de la varianza de la conducta intraemprendedora de la fuerza laboral de las Pymes, dejando en evidencia con confiabilidad estadística lo significativo de la identificación organizacional que aumenta en $17 \%$ la varianza explicada.

Respecto a la influencia individual de los constructos sobre la conducta intraemprendedora, autonomía es el que tiene un mayor impacto positivo en la IB, seguido por apoyo de la dirección, pues presentan un coeficiente estandarizado de 0.39 y 0.31 , respectivamente, tanto en el modelo base que se ilustra en la Figura 3 como en los otros modelos que se generan de las variantes del modelo planteadas anteriormente. Por lo tanto, para disponer de conductas intraemprendedoras en las empresas Pymes, sus líderes deben instalar una cultura y arquitectura organizacional que potencie la autonomía, donde el apoyo de la dirección contribuya con este propósito, reduciendo la incertidumbre para el logro de las metas en cada proceso e integralmente, incrementando la identificación organizacional, resultados consistentes con los aportes de investigaciones realizadas por Moriano et al. (2009), Barbosa (2013), Fahed-Sreih (2012), Medina et al. (2008), García et al. (2014) y, Frias y Véliz (2013). Así se conformará un escenario laboral que impulsará la contribución del capital humano en la empresa, lo que aportará a superar los desafíos urgentes y estratégicos expresados en la actual línea base de las Pymes Región de Coquimbo y Chile. 
Por otra parte, la incertidumbre en las tareas presenta influencia estadística significativa y confirma su influencia negativa, es decir, mientras mayor es la incertidumbre en las tareas, menor será la identificación con su empresa. Al relacionarla directamente con IB este factor no es significante para el modelo base. Por lo tanto, los líderes de las Pymes deben diseñar e implementar procesos operacionales y de servicios que reduzcan o eliminen los "costos" asociados a la incertidumbre, dado que la desorientación de los trabajadores daña el clima laboral y fortalece la desconfianza interna horizontal y vertical, derrochando como consecuencia el aporte del potencial de la fuerza laboral en la creación de valor agregado y competitividad de las Pymes, lo que está alineado con lo planteado por Sánchez (2014).

El tiempo disponible aparece como un factor que no tiene significancia estadística en el modelo base, tanto con identificación organizacional como relacionada directamente con la conducta intraemprendedora, por lo tanto los líderes de las Pymes de la Región de Coquimbo, deben asumir como filosofía empresarial que la reducción o carencia de tiempo disponible no explica la baja contribución del recurso humano en el desarrollo e implementación de nuevas iniciativas para la producción de un nuevo producto o servicio innovador y/o proyectos para mejorar la productividad de los procesos y/o diversificación de la empresa para aumentar su valor económico.

La asignación de recompensas para involucrar al recurso humano de las empresas de la Región de Coquimbo, es un factor que tiene significancia estadística sobre la identificación organizacional e influye de manera positiva en esta. Esto implica que la remuneración y los incentivos contribuyen a aumentar la fidelización de los trabajadores, por lo cual, planes de recompensas pertinentes a las necesidades de la fuerza laboral y con equidad interna y externa, alineados con los objetivos de la organización, motivará a los empleados a lograr un $100 \%$ de eficacia y por ende contribuirá directamente a las empresas que tienen objetivos vinculados con innovación y competitividad, lo que es consistente con lo expresado por García et al. (2012).

\section{CONCLUSIONES}

Se demostró y ratificó la contribución operativa y estratégica de la identificación organizacional de la fuerza laboral, que estimula asumir riesgos y aportar con innovación interna, interviniendo en los procesos y generación de nuevos negocios para la empresa, logrando resultados crecientes, sostenibles y sustentables.

Mientras mayor sea la identificación de los trabajadores con su empresa, mayor será la conducta intraemprendedora que estos tengan en su puesto de trabajo, impactando positiviamente en la productividad de la organización.

La identificación organizacional se ve afectada positiva y significativamente por el apoyo de la dirección, la autonomía de la fuerza laboral y el uso de recompensas, mientras que es afectada de forma negativa por la incertidumbre de los propios colaboradores. Por su parte, el tiempo disponible no influye con significancia estadística en la identificación del trabajador con su empresa.

Un trabajador al tener una conducta intraemprendedora tiene más posibilidades para potenciar y usar sus ideas cuando este dentro de su puesto de trabajo, generando emprendimientos de valor propio, contribuyendo al logro de los objetivos estratégicos de la organización.

\section{AGRADECIMIENTOS}

Los autores agradecen el apoyo entregado por la Universidad de La Serena para terminar exitosamente esta investigación.

\section{REFERENCIAS}

Ağca, V., Y. Topal y H. Kaya, Linking intrapreneurship activities to multidimensional firm performance in Turkish manufacturing firms: An empirical study, doi: 10.1007/s11365-009-0132-5, International Entrepreneurship and Management Journal, 8(1), 15-33 (2012)

Ahmedova, S., Factors for Increasing the Competitiveness of Small and Medium- Sized Enterprises (SMEs) in Bulgaria, doi: 10.1016/j.sbspro.2015.06.155, Procedia - Social and Behavioral Sciences, 195, 1104-1112 (2015)

Antoncic, B. y R. Hisrich, Intrapreneurship: Construct refinement and cross-cultural validation, doi: 10.1016/S08839026(99)00054-3, Business Venturing, 16 (5), 495-527 (2001)

Arancibia, S., M. Donoso, R. Venegas, y C. Cárdenas, Identifying Key Factors Affecting Culture of Innovation: a Case Study of Chilean Medium Mining, Journal of Technology Management \& Innovation, 10(1), 132-145 (2015) 
Barbosa, C., Liderazgo e inteligencia emocional en personas que desempeñan jefaturas en empresas de Bogotá, Universidad \& Empresa, 15(25), 87-106 (2013)

Barragán, M. y V. Ayaviri, Innovación y Emprendimiento, y su relación con el Desarrollo Local del Pueblo de Salinas de Guaranda, Provincia Bolívar, Ecuador, doi: 10.4067/S0718-07642017000600009, Inf. Tecnológica, 28(6), 71-80 (2017)

Cabana, S., F. Cortés, D. Vega y R. Cortés, Análisis de la Fidelización del Estudiante de Ingeniería con su Centro de Educación Superior: Desafíos de Gestión Educacional, doi: 10.4067/S0718-50062016000600009, Formación Universitaria, 9(6), 93-104 (2016)

Cabarcas, A., P. Puello y R. Martelo, Sistema de Información Soportado en Recuperación XML para Pequeñas y Medianas Empresas (PYME) de Cartagena de Indias, Colombia, doi: 10.4067/S0718-07642015000200016, Información Tecnológica, 26(2), 135-144 (2015)

Chaitanya, V., Motivation in the Workplace to Improve the Employee Performance, International Journal of Engineering Technology, Management and Applied Sciences, 2(6), 221-230 (2014)

Chávez, N., La auto-organización en organizaciones que aprenden, Rev. Iberoamericana de Ciencias, 1(6), 13-26 (2014)

Chiang, V., P, Núñez, M. Martín y B. Salazar, Compromiso del Trabajador hacia su Organización y la relación con el Clima Organizacional: Un Análisis de Género y Edad. Panorama Socioeconómico, 28 (40), 90-100 (2010)

Chirumbolo, A. y J. Hellegren, Individual and Organizational Consequences of Job Insecurity: A European Study, doi: 10.1177/0143831X03024002004, Economic and Industrial Democracy, 24(2), 217-240 (2003)

Demirci, A., In Pursuit of Corporate Entrepreneurship: How Employees Perceive the Role of Formalization and Centralization, doi: 10.5296/jmr.v5i3.3597, Journal of Management Research, 5(3), 115-133 (2013)

Echeverri, A., N. Lozada, y J. Arias, Incidencia de las Prácticas de Gestión del Conocimiento sobre la Creatividad Organizacional, doi: 10.4067/S0718-07642018000100071, Información Tecnológica, 29(1), 71-82 (2018)

Espinoza, M., O. Sanhueza, N. Ramírez, y K. Sáez, A validation of the construct and reliability of an emotional intelligence scale applied to nursing students, doi: 10.1590/0104-1169.3498.2535, Revista Latino-Americana de Enfermagem, 23(1), 139-147 (2015)

Fahed-Sreih, J., The effect of investing in hiring, human resource planning, and employee development on labor productivity: case of Lebanon, Journal of International Business Research, 11(1), (2012)

Fernández, F., M. López, I. Maeztu, A. Martín, El techo de vidrio de las pequeñas y medianas empresas, Revista de Estudios Empresariales, Segunda Ėpoca (1), 231-247 (2010)

Forsyth, C., y B. Mason, Shared leadership and group identification in healthcare: The leadership beliefs of clinicians working in interprofessional teams, doi: 10.1080/13561820.2017.1280005, Journal of Interprofessional Care, 31(3), 291$299(2017)$

Frias, H. y J. Véliz, La adaptabilidad a los cambios imprevistos del entorno y la creación de capacidades para el autocontrol en la empresa, Técnica Administrativa, 12(01), (2013)

García, M., N. Posada y C. Hernández, La motivación y los sistemas de recompensas y su impacto en la producción, Contribuciones a La Economía, (7), 2-18 (2012)

García, M., R. González, M. Aldrete, M. Acosta, S. León, Relación entre Calidad de Vida en el Trabajo y Síntomas de Estrés en el Personal Administrativo Universitario, doi: 10.4067/S0718-24492014000200007, Ciencia \& trabajo, 16(50), 97-102 (2014)

Gaxiola, C., S. González y Z. Contreras, Resilience Influence, Goals and Social Context in the Academic Achievement of High School Students, Revista Electrónica de Investigación Educativa, 14(1), 164-181 (2012)

Gonzalez-Mulé, E., S. Courtright, D. DeGeest, J-Y. Seong y D-S. Hong, Channeled Autonomy The Joint Effects of Autonomy and Feedback on Team Performance Through Organizational Goal Clarity, doi: 10.1177/0149206314535443, Journal of Management (2016)

Hameed, A., e I. Abdul, SEM-PLS Analysis of Inhibiting Factors of Cost Performance for Large Construction Projects in Malaysia: Perspective of Clients and Consultants, doi: 10.1155/2014/165158, The Scientific World Journal (2014)

Hernández, H., D. Cardona y J. Del Rio, Direccionamiento Estratégico: Proyección de la Innovación Tecnológica y Gestión Administrativa en las Pequeñas Empresas, doi: 10.4067/S0718-07642017000500003, Información Tecnológica, 28(5), 15-22 (2017)

Kuratko, D., J. Hornsby y J. Bishop, An Examination of Managers' Entrepreneural Actions and Job Satisfaction, The International Entrepreneurship and Management Journal, 1(3), 275-291 (2005)

Medina, A., C. Gallegos y P. Lara, Motivación y satisfacción de los trabajadores y su influencia en la creación de valor económico en la empresa, doi: 10.1590/S0034-76122008000600009, Rev. de Administração Pública, 42(6), 1213-1230 (2008)

MEFT, Ministerio de Economía Fomento y Turismo de Chile, Informe de Resultados, Empresas Chilenas (2015)

Mengual, A., D. Juárez, F. Sempere, y A. Rodríguez, La gestión del tiempo como habilidad directiva, 3Ciencias, 5-10 (2012) 
Ming, L., The Likelihood of Corporate Entrepreneurship in Large Corporations, doi: 10.9734/BJEMT/2013/4585 British Journal of Economics, Management \& Trade, 3(4), 441-451 (2013)

Ministerio de Economía, Fomento y Turismo de Chile, Dinámica Empresarial: Brechas regionales y sectoriales de las pymes en Chile (2016)

Moriano, J., G. Topa, E. Valero, y J. Lévy, Identificación organizacional y conducta "intraemprendedora", Anales de Psicología, 25(2), 277-287 (2009)

Rubio, G., Las contribuciones del intraemprendimiento a la estrategia de manufactura, doi: 10.15665/rde.v13i1.340, Dimensión Empresarial, 13(1), 95-110 (2015)

Sánchez, M., Commitment and stress on employees in Banks, doi: 10.18002/pec.v0i16/17.1336, Pecvnia: Revista de La Facultad de Ciencias Económicas y Empresariales, Universidad de León, 17, 85-100 (2014)

Sheshadri, D., y A.Tripathy, Innovation through Intrapreneurship: The Road Less Travelled, Journal of Decision Makers, 31(1), 17-29 (2006)

Simanca, M., L. Montoya y C. Bernal, Gestión del Conocimiento en Cadenas Productivas. El Caso de la Cadena Láctea en Colombia, Información Tecnológica, 27(3), 93-106 (2016)

Topa, G., y F. Morales, Identificación organizacional y proactividad personal en grupos de trabajo: Un modelo de ecuaciones estructurales Introducción, Anales de Psicología, 22(2), 234-242 (2006) 